\title{
ON A DUALITY THEOREM OF ABELIAN VARIETIES OVER HIGHER DIMENSIONAL LOCAL FIELDS
}

\author{
YOSHIHIRO KOYA
}

\begin{abstract}
In this paper, we prove a duality theorem of abelian varietıes over higher dimensional local fields under some conditions. It might be a one of generalization of the classical Tate duality theorem of abelian varietıes over local fields.
\end{abstract}

\section{Introduction}

In this paper we show a duality theorem of the Galois cohomology groups related to abelian varieties over higher dimensional local fields. It might be a good generalization of the classical Tate duality of abelian varieties over usual local fields.

In order to understand our situation, we recall the classical Tate duality. Let $K$ be a local field of characteristic 0 , that is, a complete discrete valuation field with finite residue field. Let $A$ be an abelian variety over $K$, and $A^{t}$ the dual variety of $A$ over $K$. The Weil-Barsotti formula makes the following identification (cf. [7, Théorème 6, §16, No. 17 Chapitre VII])

$$
A^{t}(K)=\operatorname{Ext}_{K}^{1}\left(A, G_{m}\right) \text {. }
$$

Then we know that the canonical pairing

$$
H^{r}(K, A) \times H^{1-r}\left(K, A^{t}\right) \rightarrow \boldsymbol{Q} / \boldsymbol{Z} \quad(r=0,1)
$$

induces isomorphisms

$$
\begin{aligned}
& H^{1}(K, A)=A^{t}(K)^{*}, \\
& H^{1}\left(K, A^{t}\right)=A(K)^{*},
\end{aligned}
$$

where $A^{t}(K)^{*}$ and $A(K)^{*}$ stand for the Pontrjagin duals of them. The proof of the fact above deeply depends on the local class field theory.

The above remark and Kato's higher dimensional local class field theory might lead us to the idea to establish the higher Tate duality. Actually one can prove a duality theorem between the torsion parts of abelian varieties over

Recelved May 28, 1999; revised December 27, 1999. 
them without any difficulty. But the point is to collect such torsion parts and to recover the duality of the whole abelian varieties.

The idea to do it is to use the motivic cohomology. Thus our generalization of the classical Tate duality is described in terms of hypercohomology. More exactly, our higher Tate duality has the following form:

THEOREM 1.1 (cf. Theorem 5.1). Let $K$ be an $N$-dimensional local field. There exists the following canonical pairing

$$
H^{r}\left(K, A^{t} \otimes^{L} \check{Z}(N-1)\right) \times H^{N-r}(K, A) \rightarrow \boldsymbol{Q} / \boldsymbol{Z}
$$

and it induces the isomorphism

$$
\left.H^{N-r}\left(K, A^{t} \otimes L \check{Z}(N-1)\right)(\text { non- } p)=\left(\widehat{H^{r}(K, A}\right)\right)^{*}(\text { non- } p)
$$

for each $r=0,1, \ldots, N$. About the notation "non- $p$ ", consult with the end of this section.

Here $\widehat{H^{r}(K, A)}$ stands for $\lim _{n} H^{r}(K, A) / n$, and $\check{Z}(m)$ means the Kahn complex which is defined by

$$
\check{\boldsymbol{Z}}(m)= \begin{cases}\boldsymbol{Z} & \text { if } m=0 ; \\ \underbrace{\boldsymbol{G}_{m} \otimes \otimes^{L} \cdots \otimes^{L} \boldsymbol{G}_{m}}_{m \text {-tımes }}[-m] & \text { if } m>0 .\end{cases}
$$

As is well-known, the complex $\check{Z}(n)$ has the following properties:

THEOREM 1.2 (Proposition 1, [1]). (1) $\check{Z}(n)$ is acyclic outside $[1, n]$.

(2) For each $m$ prime to $\operatorname{ch}(K)$ there exists a distinguished triangle:

$$
\check{Z}(n) \stackrel{m}{\rightarrow} \check{Z}(n) \rightarrow \mu_{m}^{\otimes n} \rightarrow \check{Z}(n)[1] .
$$

It might be easy to understand that our approach is one of the philosophy shown in [5].

Finally the author would like to mention some difficulties for strengthening our result. In the proof of our main theorem, we extensively enjoy the theory of the Euler-Poincare characteristic of the Galois cohomology of higher dimensional local fields with finite coefficient. In other words we use the generalized method introduced in $[6, \S 2$ and $\S 3$, Chapter I]. As in Remark 3.2, if $K$ is a 2dimensional local field with the residue field $\boldsymbol{F}_{p}((T))$, the group $H^{1}\left(K, \mu_{p}\right)$ is infinite. And we cannot use the Euler-Poincaré characteristic in such a case. This is the reason why the author cannot treat the $p$-primary torsion part.

NOTATIONS AND CONVENTIONS. In this paper, unless the contrary is explicitly stated, we employ the following notations and conventions:

(1) For an abelian group $A$, we denotes the group of $n$-torsion elements of $A$ by $A_{n}$ and sometime by $A / n$ the quotient group $A / n A$. Moreover, for a fixed prime number $p$, we denote 


$$
A(\text { non }-p)=\bigoplus_{l \neq p} A(l),
$$

where the product runs through all prime which is prime to $p$, and $A(l)$ means the $l$-primary torsion part of $A$.

(2) For an abelian group $A$, we put $A^{*}=\operatorname{Hom}(A, \boldsymbol{Q} / \boldsymbol{Z})$.

(3) Let $A, B$ and $C$ be abelian groups. If the sequence

$$
A(\text { non }-p) \rightarrow B(\text { non }-p) \rightarrow C(\text { non }-p)
$$

is exact, then we call that the sequence

$$
A \rightarrow B \rightarrow C
$$

is exact modulo $p$-primary torsion part.

(4) For a field $F$, we denote the separable closure of $F$ by $F_{s}$.

(5) For a complete discrete valuation field $K$, we denote by $K_{\mathrm{nr}}$ the maximal unramified extension field of $K$.

(6) For a finite set $M$, we denote the order of $M$ by $[M]$.

\section{Duality with finite coefficient}

In this section we establish a duality theorem of the Galois cohomology with finite coefficient, which is an easy consequence of the higher dimensional local class field theory.

In this section let $K$ be an $N$-dimensional local field, and $F$ its residue field. Then there is a sequence of fields $k=k_{0}, \ldots, k_{N-1}=F, k_{N}=K$ with the following properties:

(1) $k_{0}$ is a finite field.

(2) $k_{l}$ is a complete discrete valuation field whose residue field is $k_{l-1}$ for each $i=1, \ldots, N$.

We often mention some properties of the field $k_{0}$ above. Thus it might be convenient to call the field $k_{0}$ the final residue field of $K$.

THEOREM 2.1. Let $K$ be an $N$-dimensional local field, and $k$ its final residue field. Let $M$ be a finite $\mathrm{Gal}\left(K_{s} / K\right)$-module. Assume that the order $n$ of $M$ is prime to $\operatorname{ch}(k)=p>0$. Then the canonical map induced by the Yoneda pairing

$$
\alpha^{r}(K, M): \operatorname{Ext}_{K}^{r}\left(M, K_{s}^{\times}\right) \rightarrow H^{N+1-r}\left(K, M \otimes^{L} \check{Z}(N-1)\right)^{*}
$$

is an isomorphism for each $r=0, \ldots, N+1$.

Furthermore the order of the both groups are finite.

Proof. First of all we prove the finiteness.

By the $N$-dimensional local class field theory established by Kato ([2], [3] and [4]), we already know that there exists a canonical isomorphism

$$
H^{r}(K, Z / n Z)=\left(K_{r}^{M} K / n K_{r}^{M} K\right)^{*} .
$$


If $n$ is prime to $\operatorname{ch}(k)$, and therefore to $\operatorname{ch}(F)$, we have the isomorphism

$$
K_{r}^{M} K / n K_{r}^{M} K=K_{r}^{M} F / n K_{r}^{M} F \oplus K_{r-1}^{M} F / n K_{r-1}^{M} F
$$

for each $r=1, \ldots, N+1$. Thus it is not hard to see that this group is finite.

Now we take a finite Galois extension field $L$ of $K$ such that

(1) $L$ contains a primitive $n$-th root of 1 ;

(2) $\operatorname{Gal}\left(K_{s} / L\right)$ acts trivially on $M$.

Then we may assume that $M \otimes^{L} \check{Z}(N-1)$ is isomorphic to the direct sum of finite number of copies of $\boldsymbol{Z} / m_{i} Z$, where $m_{i}$ is a divisor of $n$, as a complex of $\operatorname{Gal}\left(K_{s} / L\right)$-module. Therefore the group $H^{r}\left(L, M \otimes^{L} \check{Z}(N-1)\right)$ is a finite group for each $r=0, \ldots, N+1$. The Hochschild-Serre spectral sequence

$$
H^{r}\left(L / K, H^{s}\left(L, M \otimes^{L} \check{Z}(N-1)\right)\right) \Rightarrow H^{r+s}\left(K, M \otimes^{L} \check{Z}(N-1)\right)
$$

proves that the group $H^{r}\left(L, M \otimes^{L} \check{Z}(N-1)\right)$ is finite for each $r$.

Next we prove that the map $\alpha^{r}(K, M)$ is an isomorphism for each $r=0, \ldots, N+1$.

Consider the induced module $R_{L / K} M$ of $M$ such that $H^{r}\left(K, R_{L / K} M\right)=$ $H^{r}(L, M)$. Then we have the following exact sequence:

$$
0 \rightarrow M \rightarrow R_{L / K} M \rightarrow N \rightarrow 0,
$$

where $N$ is the cokernel of the map $M \rightarrow R_{L / K} M$. Furthermore it should be noted that this map induces the canonical restriction map $H^{r}(K, M) \rightarrow$ $H^{r}(L, M)$.

Now assume for a while that $\alpha^{r}(L, M)$ is an isomorphism for each $r$. Consider the following commutative diagram,

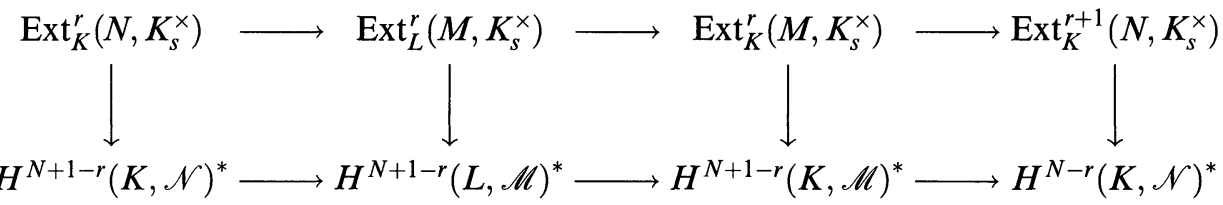

where we put $\mathscr{N}=N \otimes^{L} \check{Z}(N-1)$ and $\mathscr{M}=M \otimes^{L} \check{Z}(N-1)$. Assume that we proved that the map $\alpha^{r+1}(K, M)$ is isomorphic for any $M$, and therefore for $N$ defined above. From the five lemma we can conclude that the map $\alpha^{r}(K, M)$ is surjective. Therefore, since $M$ is assumed to be any, the map $\alpha^{r}(K, N)$ is also surjective. Hence, again by using the five lemma (or elementary diagram chasing), we can conclude that the map $\alpha^{r}(K, M)$ is injective.

Thus we can prove in the same manner that the map $\alpha^{r-1}(K, M)$ is an isomorphism.

The rest we have to do is to prove that the map $\alpha^{r}(L, M)$ is an isomorphism for each $r$. Since $\operatorname{Gal}\left(K_{s} / L\right)$ acts trivially on $M$, we may assume $M=Z / n Z$. In this case we already know $\operatorname{Ext}_{L}^{r}\left(Z / n Z, K_{s}^{\times}\right)=H^{r}\left(L, \mu_{n}\right)$. Thus we only have to show that the map

$$
\alpha^{r}(L, Z / n Z): H^{r}\left(L, \mu_{n}\right) \rightarrow H^{r}\left(L, \mu_{n}^{\otimes(N-1)}\right)^{*}
$$


is an isomorphism for every $r$. But this is nothing but the $N$-dimensional local class field theory established by Kato.

COROLlaRY 2.2. Under the conditions in Theorem 2.1, we have isomorphisms:

$$
H^{r}\left(K, M^{D}\right) \rightarrow H^{N+1-r}\left(K, M \otimes \otimes^{L} \check{Z}(N-1)\right)^{*} .
$$

Here $M^{D}=\operatorname{Hom}_{K_{s}}\left(M, K_{s}^{\times}\right)$.

Proof. Since $K_{s}^{\times}$is divisible by any integers prime to $\operatorname{ch}(F)$, we have $\operatorname{Ext}_{K_{s}}^{r}\left(M, K_{s}^{\times}\right)=0$ for all $r>0$. Therefore, from the spectral sequence:

$$
H^{r}\left(K, \operatorname{Ext}_{K_{s}}^{r}\left(M, K_{s}^{\times}\right)\right) \Rightarrow \operatorname{Ext}_{K}^{r}\left(M, K_{s}^{\times}\right),
$$

we can conclude $H^{r}\left(K, M^{D}\right)=\operatorname{Ext}_{K}^{r}\left(M, K_{s}^{\times}\right)$.

The following proposition can be easily proved by the similar method used in the proof of the main theorem in this section. This proposition might be used in the later section.

Proposition 2.3. Let $K$ be a henselian discrete valuation field with a separably closed residue field $F$. And let $M$ be a finite $\operatorname{Gal}\left(K_{s} / K\right)$-module whose order is prime to $\operatorname{ch}(F)$. Then, we have $M(K) \simeq H^{1}(K, M)$. Here the isomorphism is induced by the cup-product pairing.

Proof. Let $L$ be a finite extension field of $K$ such that the group $\operatorname{Gal}\left(L_{s} / L\right)$ acts trivially on $M$. Then, as in the proof of Theorem 2.1 , there is an exact sequence:

$$
0 \rightarrow M \rightarrow R_{L / K} M \rightarrow N \rightarrow 0,
$$

where $N$ is the cokernel of the map $M \rightarrow R_{L / K} M$, and this map induces the canonical restriction map $H^{q}(K, M) \rightarrow H^{q}(L, M)$.

Consider the following commutative diagram:

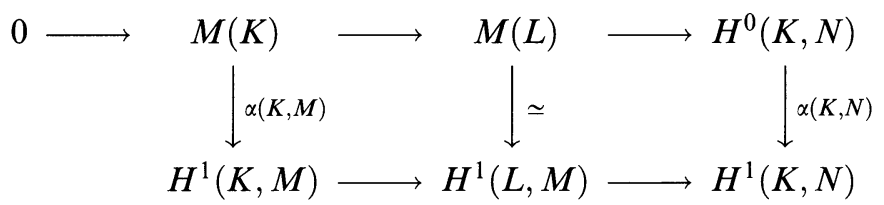

It is clear that $\alpha(K, M)$ is injective. Since $M$ is any, $\alpha(K, N)$ is also injective. Therefore, by elementary diagram chasing, we can show that the map $\alpha(K, M)$ is also surjective.

\section{The Euler-Poincaré characteristic}

In this section, unless the contrary is explicitly stated, let $K$ be an $N$ dimensional local field with residue field $F$, and $k$ the final residue field. 
In this section we define the Euler-Poincare characteristic of the Galois cohomology with finite coefficient, and calculate them under some conditions.

First of all we define the Euler-Poincaré characteristic.

Definition 3.1. Let $K$ be an $N$-dimensional local field, and $M$ a finite $\operatorname{Gal}\left(K_{s} / K\right)$-module whose order is prime to $\operatorname{ch}(k)=p>0$. Then, the EulerPoincaré characteristic $\chi(K, M)$ is defined as follows:

$$
\chi(K, M)=\sum_{l=0}^{\infty}(-1)^{l}\left[H^{l}(K, M)\right],
$$

where $\left[H^{l}(K, M)\right]$ stands for the order of the group $H^{l}(K, M)$.

Note that the above sum is actually a finite sum because the cohomological dimension of higher dimensional local fields are finite.

Remark 3.2. In the case which the order of $M$ is divided by $\operatorname{ch}(k)=p>0$, the order of the Galois cohomology group $H^{l}(K, M)$ may be infinite. Let $K$ be a 2-dimensional local field of characteristic 0 whose residue field is $F=\boldsymbol{F}_{p}((T))$, for example. Assume now $M=\mu_{p}$. In such a case the order of $H^{\imath}(K, M)$ is no longer finite. Indeed,

$$
H^{1}\left(K, \mu_{p}\right)=K^{\times} /\left(K^{\times}\right)^{p}=Z / p Z \oplus F^{\times} /\left(F^{\times}\right)^{p} \oplus U_{K}^{(1)} / p U_{K}^{(1)} .
$$

However, we have an exact sequence

$$
U_{K}^{(2)} / p U_{K}^{(2)} \rightarrow U_{K}^{(1)} / p U_{K}^{(1)} \rightarrow F \rightarrow 0 .
$$

Therefore, the group $H^{1}\left(K, \mu_{p}\right)$ cannot be finite.

This is the reason why we cannot adopt the above definition of the EulerPoincaré characteristic in such a case.

Thus in the rest of the paper we assume that the order of $M$ is prime to the characteristic of the final residue field of the field we consider.

Proposition 3.3. Let $K$ be an $N$-dimensional local field, and $M$ a finite $\operatorname{Gal}\left(K_{s} / K\right)$-module.

Then

$$
\chi(K, M)=0
$$

Proof. We prove by induction on $N$.

In the case $N=0$, that is, in the case which $K$ is a finite field, we know $\operatorname{Gal}\left(K_{s} / K\right)=\hat{Z}$. Let $\sigma$ be a generator of $\hat{Z}$. Then, we have an exact sequence

$$
0 \longrightarrow H^{0}(\hat{Z}, M) \longrightarrow M \stackrel{1-\sigma}{\longrightarrow} M \longrightarrow H^{1}(\hat{Z}, M) \rightarrow 0 .
$$

Therefore, we have $\chi(K, M)=0$. 
Assume $N>0$. Since the order of $M$ is prime to $\operatorname{ch}(k)=p>0$, we have an exact sequence

$$
0 \rightarrow H^{r}\left(F, H^{0}\left(K_{\mathrm{nr}}, M\right)\right) \rightarrow H^{r}(K, M) \rightarrow H^{r-1}\left(K, H^{1}\left(K_{\mathrm{nr}}, M\right)\right) \rightarrow 0 .
$$

Here we should note that the group $H^{r}\left(K_{\mathrm{nr}}, M\right)$ is finite for each $r=0,1$.

Thus we have

$$
\begin{aligned}
\chi(K, M) & =\sum_{l=0}^{\infty}(-1)^{l}\left[H^{l}(K, M)\right] \\
& =\sum_{l=0}^{\infty}(-1)^{l}\left[H^{l}\left(F, H^{0}\left(K_{\mathrm{nr}}, M\right)\right)\right]-\sum_{l=0}^{\infty}(-1)^{l}\left[H^{l}\left(F, H^{1}\left(K_{\mathrm{nr}}, M\right)\right)\right] \\
& =\chi\left(F, H^{0}\left(K_{\mathrm{nr}}, M\right)\right)-\chi\left(F, H^{1}\left(K_{\mathrm{nr}}, M\right)\right) .
\end{aligned}
$$

By the induction hypothesis, we can conclude $\chi(K, M)=0$.

The following proposition is an application of the result of this section.

Proposition 3.4. Let $K$ be an $N$-dimensional local field and $k$ be its final residue field of $K$. And let $T$ be an affine torus over $K$. If $n$ is a non-negative integer prime to $\operatorname{ch}(k)=p>0$, then we have $\left[T_{n}(K)\right] \leq[T(K) / n T(K)]$.

Proof. We prove the proposition by induction on $N$. Clearly the assertion holds in the case which $K$ is a finite field. Therefore, we may assume $N>0$.

From the Hochschild-Serre spectral sequence, we have an exact sequence:

$$
0 \rightarrow H^{q}\left(F, T_{n}\right) \rightarrow H^{q}\left(K, T_{n}\right) \rightarrow H^{q-1}\left(F, H^{1}\left(K_{\mathrm{nr}}, T_{n}\right)\right) \rightarrow 0 .
$$

From the above sequence, we have:

$$
\sum_{q=2}^{\infty}(-1)^{q}\left[H^{q}\left(K, T_{n}\right)\right]=\sum_{q=2}^{\infty}(-1)^{q}\left[H^{q}\left(F, T_{n}\right)\right]+\sum_{q=1}^{\infty}(-1)^{q-1}\left[H^{q}\left(F, H^{1}\left(K_{\mathrm{nr}}, T_{n}\right)\right)\right] .
$$

On the other hand, from the definition of the Euler-Poincare characteristic, we know:

$$
\sum_{q=1}^{\infty}(-1)^{q-1}\left[H^{q}\left(F, H^{1}\left(K_{\mathrm{nr}}, T_{n}\right)\right)\right]=\left[H^{0}\left(F, H^{1}\left(K_{\mathrm{nr}}, T_{n}\right)\right)\right] \geq 0 .
$$

Added to this, from the induction hypothesis, we can conclude:

$$
\sum_{q=2}^{\infty}(-1)^{q}\left[H^{q}\left(F, T_{n}\right)\right]=[T(F) / n T(F)]-\left[T_{n}(F)\right] \geq 0
$$

(Since $H^{1}(F, T)=0$, we deduce $H^{1}\left(F, T_{n}\right)=T(F) / n T(F)$ ). 
Therefore, we have shown that

$$
[T(K) / n T(K)]-\left[T_{n}(K)\right]=\sum_{q=2}^{\infty}(-1)^{q}\left[H^{q}\left(K, T_{n}\right)\right] \geq 0 .
$$

This completes the proof of the proposition.

\section{Estimations of the orders of kernel and cokernel}

In this section, unless the contrary is explicitly stated, we keep notations and conventions in the previous section. The aim of this section is to prove the following proposition.

Proposition 4.1. Let $K$ be an $N$-dimensional local field with residue field $F$, and $A$ an abelian variety over $K$. Let $n$ be a non-negative integer prime to $\operatorname{ch}(k)=p>0$. Then, for each $r=0,1, \ldots, N-1$,

$$
\left[H^{r}\left(K, A \otimes \otimes^{L} \check{Z}(N-1)\right) / n\right]=\left[H^{r}\left(K, A \otimes^{L} \check{Z}(N-1)\right)_{n}\right] .
$$

We need some lemma.

Lemma 4.2. Let $K$ be an $N$-dimensional local field. And let $m \geq N$. Then the group $H^{r}\left(K, \lim _{n} \mu_{n}^{\otimes m}\right)($ non $-p)$ is finite for each $r$.

Proof. We prove the lemma by using induction on $N$. If $N=0$, the statement is clear because $K$ is a finite field.

Thus we may assume $N>0$. From the Hochschild-Serre spectral sequence, the following sequence is exact:

$$
0 \rightarrow H^{r}\left(F, \mu_{n}^{\otimes m}\right) \rightarrow H^{r}\left(K, \mu_{n}^{\otimes m}\right) \rightarrow H^{r-1}\left(F, H^{1}\left(K_{\mathrm{nr}}, \mu_{n}^{\otimes m}\right)\right) \rightarrow 0
$$

Since $n$ is prime to the characteristic of $F$, we have $H^{1}\left(K_{\mathrm{nr}}, \mu_{n}^{\otimes m}\right)=$ $\mu_{n}^{\otimes(m-1)}\left(F_{s}\right)$. By taking limit, we obtain an exact sequence

$$
\left.0 \rightarrow H^{r}\left(F, \underline{\lim }_{n} \mu_{n}^{\otimes m}\right) \rightarrow H^{r}\left(K, \underline{\lim }_{n} \mu_{n}^{\otimes m}\right) \rightarrow H^{r-1}\left(F, \underline{\lim }_{n} \mu_{n}^{\otimes(m-1)}\right)\right) \rightarrow 0
$$

From the induction hypothesis, the both right and left groups are finite.

This completes the proof of the lemma.

LEMMA 4.3. Let $K$ be an $N$-dimensional local field with the residue field $F$. And let $A$ be an abelian variety over $K$. If for an arbitrary abelian variety $B$ over $F$ the group $H^{r}\left(F, \lim _{n} B_{n} \otimes \mu_{n}^{\otimes(N-1)}\right)$ is finite, then the group $H^{r}\left(K, \underline{\lim }_{n} A_{n} \otimes \mu_{n}^{\otimes(N-1)}\right)$ is also finite.

Proof. Since any abelian varieties have a potentially semi-abelian reduction, we may assume that $A$ has semi-abelian reduction. Added to this, since the 
connected component of $A\left(F_{s}\right)$ is of index finite, we may assume that $A\left(F_{s}\right)$ is connected. Then we may also assume that $A\left(F_{s}\right)$ is an extension of an abelian variety $B$ by an affine torus $\boldsymbol{G}_{m}^{\oplus m}$. Therefore, there is an exact sequence:

$$
0 \rightarrow \boldsymbol{G}_{m}^{\oplus m} \rightarrow A \rightarrow B \rightarrow 0
$$

and therefore

$$
\begin{aligned}
\cdots \rightarrow H^{r}\left(F, \underline{\lim }_{n} \mu_{n}^{\otimes N}\right) & \rightarrow H^{r}\left(F,{\left.\lim _{n} A_{n} \otimes \mu_{n}^{\otimes(N-1)}\right)} \rightarrow H^{r}\left(F,{\lim _{n}}_{B_{n}} \otimes \mu_{n}^{\otimes(N-1)}\right) \rightarrow \cdots .\right.
\end{aligned}
$$

From the assumptions of the lemma and Lemma 4.2, we can deduce that the group $H^{r}\left(F, \lim _{n} A_{n} \otimes \mu_{n}^{\otimes(N-1)}\right)$ is also finite.

Consider the Hochschild-Serre spectral sequence

$$
H^{p}\left(F, H^{q}\left(K_{\mathrm{nr}}, A_{n} \otimes \mu_{n}^{\otimes(N-1)}\right)\right) \Rightarrow H^{p+q}\left(K, A_{n} \otimes \mu_{n}^{\otimes(N-1)}\right) .
$$

From this we have

$$
\begin{aligned}
0 \rightarrow H^{q}\left(F, A_{n} \otimes \mu_{n}^{\otimes(N-1)}\right) & \rightarrow H^{q}\left(K, A_{n} \otimes \mu_{n}^{\otimes(N-1)}\right) \\
& \rightarrow H^{q-1}\left(F, H^{1}\left(K_{\mathrm{nr}}, A_{n}\right) \otimes \mu_{n}^{\otimes(N-1)}\right) \rightarrow 0 .
\end{aligned}
$$

Since $H^{1}\left(K_{\mathrm{nr}}, A_{n}\right)=A_{n}\left(K_{\mathrm{nr}}\right)$ (cf. Proposition 2.3), the limits of the both right and left groups are finite. This completes the proof of the lemma.

Lemma 4.4. Let $K$ be a henselian discrete valuation field with a separably closed residue field $F$. Then we have

$$
H^{r}(K, A \otimes L \check{Z}(N-1))(\text { non- } p)=\underline{\lim }_{n} A_{n}(K) \otimes \mu_{n}^{\otimes m}(K)
$$

for $r=0,1$.

Proof. From the hypercohomology spectral sequence

$$
H^{p}\left(K, \mathscr{H}^{q}\left(A \otimes^{L} \check{\boldsymbol{Z}}(N-1)\right)\right) \Rightarrow H^{p+q}\left(K, A \otimes^{L} \check{\boldsymbol{Z}}(N-1)\right),
$$

we have

$$
H^{0}\left(K, A \otimes^{L} \check{\boldsymbol{Z}}(N-1)\right)=\mathscr{H}^{0}\left(A \otimes^{L} \check{\boldsymbol{Z}}(N-1)\right)^{\mathrm{Gal}\left(K_{s} / K\right)} .
$$

On the other hand, from the universal coefficient theorem

$$
\begin{aligned}
0 \rightarrow \bigoplus_{p+q=0} \mathscr{H}^{p}(A) \otimes \mathscr{H}^{q}(\check{\boldsymbol{Z}}(N-1)) & \rightarrow \mathscr{H}^{0}\left(A \otimes^{L} \check{\boldsymbol{Z}}(N-1)\right) \\
& \rightarrow \bigoplus_{p+q=1} \operatorname{Tor}_{\boldsymbol{Z}}\left(\mathscr{H}^{p}(A), \mathscr{H}^{q}(\check{\boldsymbol{Z}}(N-1))\right) \rightarrow 0
\end{aligned}
$$

and the properties of the Kahn complex, we observe

$$
\mathscr{H}^{0}\left(A \otimes \otimes^{L} \check{Z}(N-1)\right)=\operatorname{Tor}_{\boldsymbol{Z}}\left(A\left(K_{s}\right), \mathscr{H}^{1}(\check{Z}(N-1))\right) .
$$


Therefore, we have

$$
\mathscr{H}^{0}\left(A \otimes{ }^{L} \check{Z}(N-1)\right)^{\operatorname{Gal}\left(K_{s} / K\right)}=\operatorname{Tor}_{\boldsymbol{Z}}\left(A(K), \mathscr{H}^{1}(\check{Z}(N-1))^{\mathrm{Gal}\left(K_{s} / K\right)}\right) .
$$

From the fact that the right side is divisible and the Kummer theory of the Kahn complex, we obtain an exact sequence:

$$
\begin{aligned}
0 & \longrightarrow H^{r}\left(K, A_{n} \otimes \mu_{n}^{\otimes(N-1)}\right) \\
& \longrightarrow H^{r}\left(K, A \otimes \otimes^{L} \check{Z}(N-1)\right) \stackrel{n}{\longrightarrow} H^{r}\left(K, A \otimes^{L} \check{Z}(N-1)\right) \longrightarrow 0,
\end{aligned}
$$

for $r=0,1$. By taking limit and applying Lemma 2.3, we obtain our desired result.

We prove the proposition by induction on $N$. If $N=1$, the assertion is classical. Thus we may assume $N>1$. From the Hochschild-Serre spectral sequence

$$
H^{p}\left(F, H^{q}\left(K_{\mathrm{nr}}, A \otimes^{L} \check{Z}(N-1)\right)\right) \quad \Rightarrow \quad H^{p+q}\left(K, A \otimes \otimes^{L} \check{Z}(N-1)\right)
$$

and the previous lemma, we can deduce that the sequence

$$
\begin{aligned}
0 \rightarrow H^{q}\left(F, \underline{\lim }_{n} A_{n} \otimes \mu_{n}^{\otimes(N-1)}\right) & \rightarrow H^{q}\left(K, A \otimes^{L} \check{Z}(N-1)\right) \\
& \rightarrow H^{q-1}\left(F, \underline{\lim }_{n} A_{n} \otimes \mu_{n}^{\otimes(N-1)}\right) \rightarrow 0
\end{aligned}
$$

is exact modulo p-primary torsion part. From Lemma 4.3, both the right and left groups is finite for $q \leq N-1$. Therefore, the middle group is also finite. This completes the proof of the proposition.

\section{The higher Tate duality}

In this section, unless the contrary is explicitly stated, we keep our notations and conventions in the previous section. Added to this, for any abelian group $A$, we put

$$
\widehat{A}=\lim _{n} A / n A
$$

In this section we prove our main theorem:

THEOREM 5.1. The canonical pairing

$$
H^{r}\left(K, A^{t} \otimes{ }^{L} \check{Z}(N-1)\right) \times H^{N-r}(K, A) \rightarrow \boldsymbol{Q} / \boldsymbol{Z}
$$

induces the isomorphism

$$
\left.H^{N-r}\left(K, A^{t} \otimes^{L} \check{Z}(N-1)\right)(\text { non- } p)=\left(\widehat{H^{r}(K, A}\right)\right)^{*}(\text { non- } p)
$$

for each $r=0,1, \ldots, N$. 
In the rest of the paper we put

$$
\begin{gathered}
A^{t}(N-1)=A^{t} \otimes^{L} \check{Z}(N-1) \\
\mathscr{M}=A_{n}^{t} \otimes \mu_{n}^{\otimes(N-1)} .
\end{gathered}
$$

Let $n$ be a positive integer prime to $\operatorname{ch}(k)=p>0$. Consider the following commutative diagram:

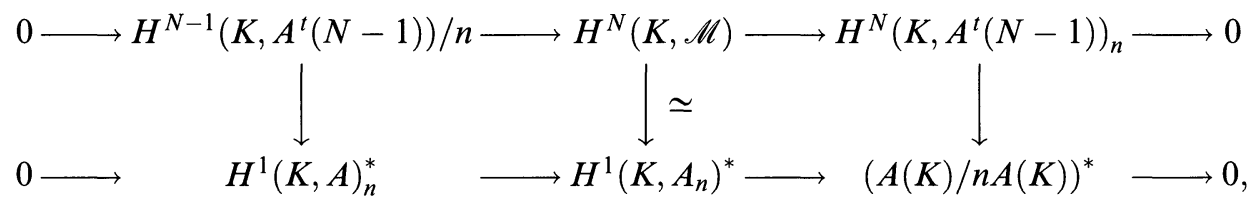

where the middle vertical arrow is an isomorphism (Corollary 2.2), and all horizontal sequences are exact. By using the snake lemma, we see that the map

$$
H^{N}\left(K, A^{t}(N-1)\right)(\text { non- } p) \rightarrow(T A(K))^{*}(\text { non- } p)
$$

is surjective. In order to show that the map is injective, we only have to show that the map

$$
H^{N}\left(K, A^{t}(N-1)\right)_{n} \rightarrow(A(K) / n A(K))^{*}
$$

is injective for each $n$ prime to $p$. But, we already know that the map is surjective. Thus, we may compare the order of the both. By easy calculation, we know

$$
\begin{aligned}
\chi(K, \mathscr{M})= & \sum_{l=0}^{\infty}(-1)^{l}\left[H^{l}(K, \mathscr{M})\right] \\
= & {\left[H^{0}\left(K, A^{t}(N-1)\right)_{n}\right] } \\
& +(-1)^{1}\left(\left[H^{0}\left(K, A^{t}(N-1)\right) / n\right]+\left[H^{1}\left(K, A^{t}(N-1)\right)_{n}\right]\right) \\
& \cdots \\
& +(-1)^{N}\left(\left[H^{N-1}\left(K, A^{t}(N-1)\right) / n\right]+\left[H^{N}\left(K, A^{t}(N-1)\right)_{n}\right]\right) \\
& +(-1)^{N+1}\left[H^{N+1}(K, \mathscr{M})\right]
\end{aligned}
$$

Since $\left[H^{r}\left(K, A^{t}(N-1)\right) / n\right]=\left[H^{r}\left(K, A^{t}(N-1)\right)_{n}\right]$ (Proposition 4.1), we have

$$
\chi(K, \mathscr{M})=(-1)^{N}\left[H^{N}\left(K, A^{t}(N-1)\right)_{n}\right]+(-1)^{N+1}\left[H^{N+1}(K, \mathscr{M})\right] .
$$

From Proposition 3.3, we can conclude

$$
\left[H^{N}\left(K, A^{t}(N-1)\right)_{n}\right]=\left[H^{N+1}(K, \mathscr{M})\right]=\left[H^{0}\left(K, A_{n}\right)\right]
$$

If we show $\left[A_{n}(K)\right] \leq[A(K) / n]$, we can conclude that the map

$$
H^{N}\left(K, A^{t}(N-1)\right)(\text { non- } p) \rightarrow(\widehat{A(K)})^{*}(\text { non- } p)
$$

is an isomorphism. Thus the rest task to do is to prove the following lemma: 
Lemma 5.2. Let $K$ be an $N$-dimensional local field. And let $A$ be an abelian variety over $K$. Then we can find a finite extension field $L$ of $K$ such that $\left[A_{n}(L)\right] \leq[A(L) / n]$ for an arbitrary non-negative integer $n$ prime to $\operatorname{ch}(k)$.

Proof. If $N=0$, the assertion is trivial. Thus, we may assume $N>0$. Since any abelian variety has potentially semi-abelian reduction, we can take a finite extension $L$ such that $A(E)$ may be an extension of an abelian variety by an affine torus, where $E$ is the residue field of $L$. Therefore, we have $[A(E) / n] \geq$ $\left[A_{n}(E)\right]=\left[A_{n}(L)\right]$ (Proposition 3.4). Here we also use the induction hypothesis. From the theory of Néron model, the canonical reduction map $A(L) \rightarrow A(E)$ is surjective. Hence, we have $[A(L) / n] \geq[A(E) / n]$.

Note that the fact that the map

$$
H^{N}\left(L, A^{t}(N-1)\right)(\text { non- } p) \rightarrow \widehat{(A(L)}^{*}(\text { non- } p)
$$

is an isomorphism also allows us to deduce $\left[A_{n}(L)\right]=[A(L) / n]$.

Now let $A_{\text {tor }}$ be the torsion part of $A$. From the above argument, we can deduce that the group $A(L) / A_{\text {tor }}(L)$ is $n$-divisible. On the other hand, let $Q$ be the cokernel of the canonical map $A(K) \rightarrow A(L)$. Then $Q / Q_{\text {tor }}$ is also $n$-divisible because of the surjectivity of the map $A(L) \rightarrow Q$. Therefore $[Q / n Q]=\left[Q_{\text {tor }} / n Q_{\text {tor }}\right]$. Since the group $Q_{\text {tor }}$ is finite, we also have $\left[A_{n}(K)\right]=$ $[A(K) / n A(K)]$. After all, we can conclude that the map

$$
H^{N}\left(K, A^{t}(N-1)\right)(\text { non- } p) \rightarrow(\widehat{A(K)})^{*}(\text { non- } p)
$$

is also an isomorphism.

The rest can be proved inductively by using Proposition 4.1.

\section{REFERENCES}

[1] B. KAHN, The decomposable part of motıvic cohomology and bijectivity of the norm residue homomorphism, Contemp. Math., 126 (1992), 79-87

[2] K. Kato, A generalization of local class field theory by using $K$-groups I, J. Fac. Sci. Univ. Tokyo Sect. IA Math., 26 (1979), 303-376.

[ 3 ] K. Kato, A generalization of local class field theory by using $K$-groups II, J. Fac. Sci. Univ. Tokyo Sect. IA Math., 27 (1980), 603-683.

[4] K. Kato, Galos cohomology of complete discrete valuation fields, Algebraic $K$-Theory, Lecture. Note in Math., 967, Sprınger-Verlag, (1980), 215-238.

[ 5] Y. KoYA, A generalization of class formation by using hypercohomology, Invent. Math., 101 (1990), 705-715.

[6] J. S. MiLne, Arithmetic Duality Theorems, Academic Press, 1986.

[7] J.-P Serre, Groupes Algébrıques et Corps de Classes, Hermann, Parıs, 1959.

Department of Mathematical Sciences

YOKOHAMA CiTy UNIVERSITY

YOKOHAMA 236-0027, JAPAN

e-mail: koya@math.yokohama-cu.ac.jp 